\title{
Advanced Region Detection Technique to Enhance Accurate Abnormality Isolation of Cancer in Digital Images
}

\author{
N. M. Mallika ${ }^{1}$, S. Janarthanam ${ }^{2}$ and A. Aruljothi ${ }^{3}$ \\ ${ }^{1}$ Assistant Professor, Department of Computer Science, Sri Vasavi College of Arts \& Science, Tamil Nadu, India \\ ${ }^{2}$ Assistant Professor, Department of Computer Science, Gobi Arts \& Science College, Tamil Nadu, India \\ ${ }^{3}$ Head \& Assistant Professor, Department of Information Technology, Gobi Arts \& Science College, Tamil Nadu, India \\ E-Mail: mallika@gmai.com,professorjana@gmail.com
}

\begin{abstract}
In recent years, extensive research is carried out in computer assisted interpretation carried out for cancer classification. Computer aided Interpretations are involves with pre-processing, contrast enhancement, segmentation, appropriate feature extraction and classification. Though considerable research is carried out in developing contrast enhancement and image segmentation techniques, cancer regions could not be isolated and extracted efficiently. Hence this work focuses on developing efficient image segmentation techniques for isolating the cancer region and also identifying suitable descriptors for describing the cancer region. Hence this work focuses to introduce a simple and easy approach for detection of cancerous tissues in mammals. Detection phase is followed by segmentation of the region in an image. Our approach uses simple image processing techniques such as averaging and thresholding along with a Max-Mean and LeastVariance technique for cancer detection. Experimental results demonstrate the effectiveness of our approach.

Keywords: Canny Operator, Cancer Detection, Edge Identification, Image- Segmentation, Media Filtering, Window Mapping
\end{abstract}

\section{INTRODUCTION}

THEcancer is the main leading cause of death in the recent years in the world. It is observed that early detection of malignancy can help in the diagnosis of the disease and it can help strongly to enhance the expectancy of survival. For the detection of cancer, various techniques are used in which the most promising technique and used by radiologist frequently. In this work X-ray CT images are taken for experimental processing .The CT images are usually of low contrast and noisy. In the images bright regions represent cancer. In some CT images, malignant tissues and normal dense tissues both may be present. To contrast between malignant and normal dense tissues is not possible only through applying thresholding [1].

Understanding the information of mass regions of cancerous lesions in an image is necessary and helps to identify the tumor and its segmentation. Therefore, detection of cancerous lesions in CT images becomes an active research area [12, 15]. Many techniques including computer-aided detection systems and intensity-based methods were introduced for the cancer segmentation in X-ray CT images. However, no solution is best promising or able to satisfy detection criteria of only including cancerous regions successfully.

\section{IMAGE SEGMENTATION}

Edge detection produces global edges in an image. This means that there is no object definition attached to the edges. Therefore it is required to somehow define the objects first and then obtain edges from them. This can be achieved by using image segmentation. The main goal of image segmentation is to divide an image into parts that have a strong correlation with objects or areas of the real world depicted in the image. This technique or group of techniques refers to dividing images into regions with similar attributes [2]. The attributes are often gray levels, colors, edges, texture characteristics, or spectral properties. The main goal of image segmentation is to simplify the representation of an image into something that is more meaningful, thus making an image easier to analyze in the image processing tasks.

\section{A. Visualization of Segments}

This process of image segmentation is one of the main parts in various applications in diagnostics. Algorithms for image segmentation are used for detecting micro calcification in CT images [3]. Whose major role is in the opportune detection and treatment. [9] created automated computed tools for micro calcification detection based on wavelet filters and used artificial neural networks. For tumor region extraction and enhancement of the classification of cancer images, edge detection operators are considered [4]. In order to segment dense areas of the abnormal isolation and the existence of mass and to visualize than other regions, the graph cuts (GC) segmentation technique is used [10] Proposed a probabilistic approach for boundary extraction in the CT images[1].

Detection of micro calcification is, in most cases, performed with preprocessed images [5] filtered images by different kinds of filters with the aim of creating a huge dictionary database. New images were compared with a database where every pixel of the image is a center of the patch. The results are probability images where a higher brightness pixel corresponds to more reliance to be micro calcification.

The final result of the segmentation process is a uniform region. According to [8], the segmentation quality is rated 
by the ratio of uniformity and homogeneity of the estimated region. The regions need to be shell-free and edges of regions are smooth and space accurate [16].

\section{B. Contribution of our Work}

To overcome the limitation, it is necessary to develop an approach to segment malignant regions properly. This inspired us to work on the problem of cancer detection in Xray images which are able to segment cancerous region along with detection. Consumption of time in execution is also important to provide good results in real time. Having this factor in mind, we develop an important and significant method which first detects the cancerous region and then segment the area covered by malignant tissues. In this paper, we are focusing onto detecting the malignant tissues which represent higher intensity values compared to background information and other regions of the affected parts.

However, in case of some normal dense tissues having similar intensities to tumor region, it is necessary to detect tumor region excluding those regions successfully. The propose a method including detection followed by segmentation of cancer region in images based on simple image processing techniques which provide good results in real time. Our method consists of two main steps (1) detection and (2) segmentation. In the detection phase, an averaging filter and thresholding operation is applied on original input image which outputs malignant region area. To find the malignant tissues, we create a rectangular window around the outputted region area and apply MaxMean and Least-Variance technique.

\section{Related Work}

The Proposed technique is based on the Fisher information measure. Bethapudi et al., proposed a detection and identification method of mass structure in digital images [14] which detect malignant tissues in following steps: (1) Thresholding to remove the background information, (2) Apply median filter for random noise removal, (3) Extract the binary image contours [7]. Thereafter, morphological open and close operations to fill the gaps in holes inside the image region. Authors proposed methodology to identify the shape of mass. Basheer et al., proposed a breast mass segmentation method based on adaptive median filtering and texture analysis [11] used adaptive median filtering for contouring the image. Thereafter, best contour is chosen based on the texture properties of the resulting Region-ofInterest (ROI).

Sampaio et al., presented a computational methodology for detection of masses in the digital images [17] can be described in following steps: (1) removing noise and objects outside the boundary and highlighting the internal structures of the breast, (2) regions containing mass are segmented using cellular neural network, (3) Thereafter the shape of these regions are analyzed through shape descriptors, (4) classification of candidate region is classified as masses or non-masses through Support Vector Machine.

In classification step, some features give geometrical information and remaining shape parameters. Computed features for each ROI are used as inputs to a supervised neural network [20]. Decision of selected ROI is pathological or not depends upon the probability provided by output neuron. Sheshadri et al., introduced the use of functions for computing texture based on statistical measures [6]. Authors employed MPM (Maximizer of the posterior margins) to perform segmentations and evaluate the region properties [3], proposed segmentation method with pectoral muscle suppression on $\mathrm{X}$ ray $\mathrm{CT}$ images. Authors of this method emphasized to split the image into interesting regions to achieve optimal search for abnormalities. Guliato et al., proposed a segmentation method of a tumor using fuzzy sets [4]. Authors proposed two segmentation methods using a fuzzy set which determines the boundary of a tumor by region growing.

\section{PROPOSED METHOD}

Expectation maximization algorithm is widely used when the data set contains missing or hidden values. This is due to the problems in the observations or collection of data's. All kind of biological analysis is carried on the data's obtained with limited test only. Expectation maximization algorithm involves in guessing an initial value and increasing the probability of acceptance of that initial parameter [13]. This is done in an iterative way. Maximization step is used to evaluate the parameter and if it is not good then discarded and a new guess is done. In contrast to the conventional techniques, in the improved EM, the mean of the missing value is estimated. This kind of statistical estimation helps in reducing the errors which occur in Markov models.

The proposed techniques introduce a simple and efficient approach to detect the cancer region in CT images. Our approach also segments the cancer region on input image. The outline of our method is described in Fig. (1)Describe the proposed method in following step.

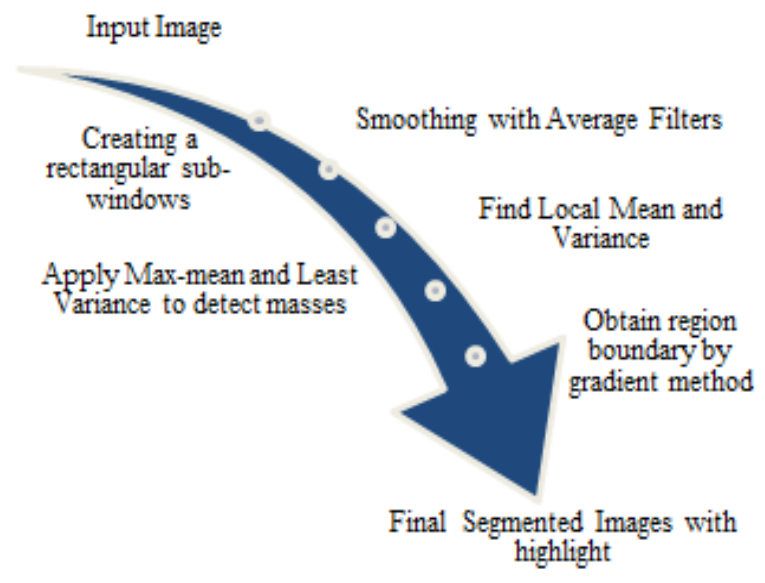

Fig. 1 The proposed method 
To take a $\mathrm{M} \times \mathrm{N}$ image $\mathrm{I}$ as input and perform averaging filter of size $s \times s$ to smooth pixels which are having intensity values similar to pixels belonging to cancer region in I, shown in Fig. (2).A thresholding parameter d is used to separate pixels of cancer region from normal region get a white patch after thresholding covering cancer region, shown in Fig.2. However it still left some pixels belonging to cancer region.

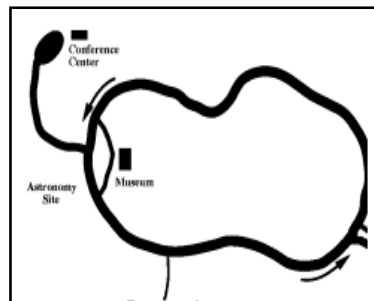

(a) Map

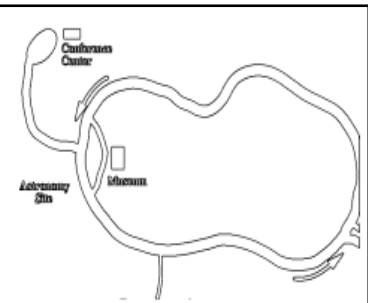

(b) Boundary
Fig. 2 The result of the boundary extracted using the proposed methods

This segmentation algorithm by merging the image domain and the feature (range) space into a joint spatial-range domain of dimension $d=p+2$, where $p$ is the dimension of the range domain. This gives an added advantage of considering both the spaces together and gives good results in cases where non-uniform illumination produces false contours when the previous segmentation algorithm is used. Therefore, the new algorithm is particularly useful to segment regions in cancers images with objects. The morphological edge detection on a user selected binary region, which is a combination of one or more segments. All the edge pixels are then considered as sample points around objects of interest. These sample points can be used then to compute the skeleton and the boundary of the object.

The steps involved improving the accuracy detection of the isolation to significantly reduce the quantity of data to be processed within image, the edge representation will retain essential information regarding the shapes of objects in the scene. To extract the exact edge line with good orientation is the major property of the edge detection technique. Calculate the upper and lower threshold values of the Enhanced image

1. Adjust the mean value based on the threshold values

2. Evaluate the no of clusters

3. Update the cluster mean value

4. Based on the clusters, the abnormality is segmented.

Global Thresholding can beused, when the pixel values of the components and that of background are fairly consistent in their respective values over the entire image.

In order to reduce the computational complexity modified energy algorithm is used for segmenting the abnormality. It's mainly depends on the success of the estimating the threshold value which separates the two homogenous region of the foreground and background of an image [18]. This function is a combination of two forces namely internal and external. The internal force is calculated from the shape of the region of interest and the external force is calculated from a higher knowledge about that image. The energy function focuses on three components namely Curvature, Continuity and image gradient. If the edges are smooth then the curvature value should be kept high. In contrast to the conventional algorithm, are initially generated by using the histograms [19]. Steps involved in the proposed algorithm are as follows: Initially from the color image, the mask image is generated. After generating the mask image, it is applied on the original image and the dissimilarity matrix is generated.

\section{EXPERIMENTAL RESULTS}

Initially, the input image is doubled and converted to grayscale (or) a binary image. The gradient is the change in the intensity with direction. The gradient of grayscale image is obtained by using appropriate masks. Further, the magnitude of the gradient is calculated by $|G|=\left|G_{x}\right|+|G y|$. Finally, the input image is directed by threshold, which identifies the pixel location as edges. After a threshold value adjusts itself to reflect the actual abnormality region.

In this work, part is segmented from the original image which is containing cancer. Further classification is performed on that part after applying ROI. The result of image segmentation is a set of segments that collectively cover the entire image, or a set of contours extracted from the image. Complete segmentation process to get segmented part is shown in Fig 3.The region of interest is extracted by mapping the coordinates of pixels in segmented part in above step to that of the original input image. This will extract the infected part from the original image and then feature extraction.

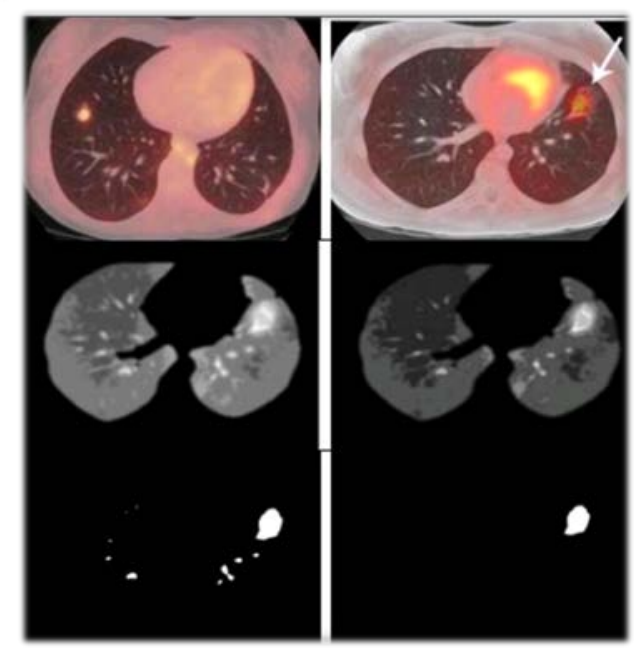

Fig. 3 Complete steps during Detection

The following calculation provide the statistical estimation and the proposed method tested on several cancer images on a desktop with IntelCore2Quad Q9550 2.83 GHz CPU with 4GB RAM. The Table I and II show results on images in which approximate time $4.20 \mathrm{sec}$ is taken to perform experiment on each image. 
TABLE I ORIGINAL IMAGE REgION AND ACCURACY ISOLATION

\begin{tabular}{|c|c|c|c|c|c|}
\hline Image & Major axis length & Perimeter & Centroid & Equiv diameter & Minor axis length \\
\hline 1.JPG & 183.206 & 587 & 209.2732 & 134.2819 & 101.6591 \\
\hline 2.JPG & 177.79 & 665 & 172.1331 & 131.1349 & 100.5351 \\
\hline 3.JPG & 756.765 & 2230 & 115.5615 & 116.4146 & 438.6437 \\
\hline 4.JPG & 760.961 & 2230 & 117.6051 & 115.3157 & 443.0261 \\
\hline 5.JPG & 765.126 & 2220 & 115.4141 & 118.006 & 445.1632 \\
\hline
\end{tabular}

TABle Ii OutPut Of Proposed TeChNiQue

\begin{tabular}{|c|c|c|c|c|c|}
\hline Image & Major Axis length & Perimeter & Centroid & Equiv Diameter & Minor Axis Length \\
\hline 1.JPG & 381.1786 & 1130 & 57.1736 & 59.516 & 217.9055 \\
\hline 2.JPG & 96.0545 & 269 & 85.8852 & 74.7631 & 59.6162 \\
\hline 3.JPG & 379.1589 & 1110 & 58.3153 & 58.7949 & 219.7077 \\
\hline 4.JPG & 381.3371 & 1110 & 59.9016 & 59.1081 & 223.1942 \\
\hline 5.JPG & 384.9321 & 1180 & 73.5965 & 65.2414 & 246.2714 \\
\hline
\end{tabular}

Comparing the last column of table I and table II, the Sensitivity is calculated for exemplars obtained from segmentation techniques. Relationship between the desired and the actual values are shown in Figure 4. From the Figure 4, it is found that the sensitivity is cenpersent for both the segmentation techniques.

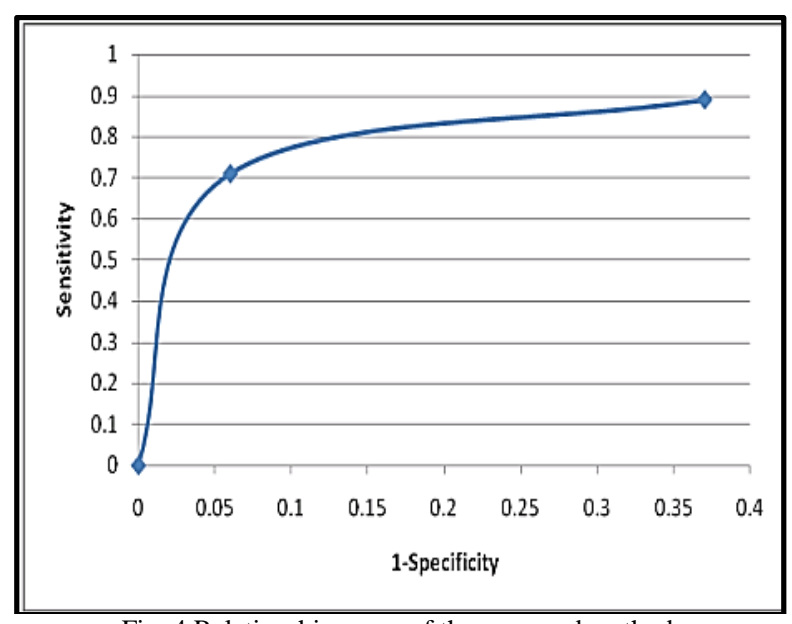

Fig. 4 Relationship curve of the proposed method

\section{CONCLUSION}

Through visual analysis, it is clear that our method is successful in segmenting the cancer region. Along with segmentation, pixels of cancer region are also identified. Our method is simple and fast because of using basic image processing techniques.

Our method can also be helpful in other medical imaging applications, pattern matching, and feature extraction. The main drawback of our method is the manual selection of threshold parameter and size of averaging filter. The Region and boundary descriptors are used for quantitatively characterizing the abnormality from the digital images.

\section{REFERENCES}

[1] H. H. Aghdam, D. Puig and A. Solanas, “A probabilistic approach for breast boundary extraction in mammograms", Computational and Mathematical Methods in Medicine, pp. 1-19, 2013.

[2] American Cancer Society, Cancer Facts, Figures, 2009-2016. [Online] Available: http://www.cancer.org.

[3] David Raba, Arnau Oliver, Joan Mart'1, Marta Peracaula and Joan Espunya, "Breast Segmentation with Pectoral Muscle Suppression on Digital Mammograms", In Pattern Recognition and Image Analysis, Springer, pp. 471-478, 2005.

[4] Denise Guliato, Rangaraj M. Rangayyan, Walter A. Carnielli, J. E. Leo Desautels, et al., "Segmentation of Breast Tumors in Mammograms using Fuzzy Sets”, Journal of Electronic Imaging, Vol. 12, No. 3, pp. 369-378, 2003.

[5] Y. Y. Dian, S. H. Fang, and C. H. Yao, "Change detection for highresolution images using multilevel segment method", Journal of Remote Sensing (in China), Vol. 20, pp. 129-137, 2016.

[6] Imran Ali, Waseem.A. Wani and Kishore Saleem, "Cancer Scenario in India with Future Perspectives,” Cancer Therapy, Vol. 8, pp. 5670, 2011.

[7] S. Janarthanam and S. Sukumaran, "Semi Supervised Soft Label Propagation Algorithmfor CBIR", 10th International Conference on Intelligent Systems and Control (ISCO 2016), Vol. 1, pp. 523-527, 2016.

[8] S. Janarthanam, et al., "Active Salient Component Classifier System on Local Features for Image Retrieval”, Indian Journal of Science and Technology, Vol. 10, No. 26, 2017.

[9] M. Kallergi, "Computer-aided diagnosis of mammographic microcalcification clusters”, Medical Physics, Vol. 31, No. 2, pp. 314-326, 2004.

[10] Mir Rayat Imtiaz Hossain, Imran Ahmed and Md. Hasanul Kabir, "Automatic Lung Cancer Detection using GLCM features”, Asian Conference on Computer Vision ACCV’14, Singapore, 2014.

[11] Nasseer M. Basheer and Mustafa H. Mohammed, "Segmentation of Breast Masses in Digital Mammograms using Adaptive Median Filtering and Texture Analysis”, Int. J. Recent Technol. Eng.(IJRTE), Vol. 2, No. 1, pp. 2277-3878, 2013.

[12] A. Oliver, A. Torrent, X. Lladó et al. “Automatic microcalcification and cluster detection for digital and digitised mammograms”, Knowledge-Based Systems, Vol. 28, pp. 68-75, 2012.

[13] B. Padmapriya, T. Kesavamurthi and H. W. Ferose, "Edge based image segmentation technique for detection and estimation of the bladder wall thickness”, Procedia Engineering, Vol. 30, pp. 828-835, 2012. 
[14] E. Prakash Bethapudi, Sreenivasa Reddy and Y. Srinivas, "Detection and Identification of Mass Structure in Digital Mammogram, Methodology”, Vol. 78, No. 14, 2013.

[15] G. ShapiroL. and G. C. Stockman, “Computer Vision”, Prentice-Hall Inc. New Jersey, pp. 279-325, 2001.

[16] K.A.G, Udeshani, R.G.N. Meegama and T.G.I. Fernando, "Statistical Feature- based Neural Network Approach for the Detection of Lung Cancer inChest X-Ray Images”, International Journal of Image Processing (IJIP , Vol. 5, No. 4, pp. 425-434, 2011

[17] Wener Borges Sampaio, Edgar MoraesDiniz, Arist 'ofanesCorrea Silva, Anselmo Cardoso De Paiva and Marcelo Gattass, "Detection of Masses in Mammogram Images using cnn, Geostatistic Functions and svm”, Computers in Biology and Medicine, Vol. 41, No. 8, pp. 653664, 2011.

[18] Z. A. Abo-Eleneen and Gamil Abdel-Azim, "A Novel Statistical Approach for Detection of Suspicious Regions in Digital Mammogram”, Journal of the Egyptian Mathematical Society, Vol. 21, No. 2, pp. 162-168, 2013.

[19] X H. Zhang, "Improving Canny operator based on CT image of the brain”, Journal of Guizhou Normal University (Natural Sciences), Vol. 34, No. 2, pp. 80-100, 2016.

[20] Zheng et al., "Image segmentation based on adaptiveK-means algorithm”, EURASIP Journal on Image and Video Processing, Vol. 68, pp. 1-10, 2018. 